\title{
Optimization of gas injection conditions during deposition of AIN layers by novel reactive GIMS method*
}

\author{
KRZYSZTOF ZDUNEK $^{1,2 \dagger}$, KATARZYNA NOWAKOWSKA-LANGIER ${ }^{2}$, RAFAL CHODUN ${ }^{1}$, \\ JERZY DORA ${ }^{3}$, SEBASTIAN OKRASA ${ }^{1}$, EWA TALIK ${ }^{4}$ \\ ${ }^{1}$ Faculty of Materials Science, Warsaw University of Technology, Warsaw, Poland \\ ${ }^{2}$ National Centre for Nuclear Research, Swierk/Otwock, Poland \\ ${ }^{3}$ Dora Power Systems, Wilczyce/Wroclaw, Poland \\ ${ }^{4}$ Institute of Physics, Silesian University, Katowice, Poland
}

\begin{abstract}
In 2011, we proposed a novel magnetron sputtering method. It involved the use of pulsed injection of working gas for the initiation and control of gas discharge during reactive sputtering of an AlN layer (Gas Injection Magnetron Sputtering - GIMS). Unfortunately, the presence of Al-Al bonds was found in XPS spectra of the AlN layers deposited by GIMS onto Si substrate. Our studies reported in this paper proved that the synchronization of time duration of the pulses of both gas injection and applied voltage, resulted in the elimination of Al-Al bonds in the AlN layer material, which was confirmed by the XPS studies. In our opinion the most probable reason of $\mathrm{Al}-\mathrm{Al}$ bonds in the AlN layers deposited by the GIMS was the self-sputtering of the Al target in the final stage of the pulsed discharge.
\end{abstract}

Keywords: plasma surface engineering; magnetron sputtering; aluminum nitride deposition

(C) Wroclaw University of Technology.

\section{Introduction}

In the last AEPSE'2011 Conference [1,2] we presented, for the first time, the concept as well as the preliminary results of the use of working gas injection to control the generation of pulsed plasma. The problem was discussed on the example of two methods of plasma surface engineering: Impulse Plasma Deposition (IPD) [3] and Pulse Magnetron Sputtering (PMS) [4-6]. Unexpectedly good properties of the layers deposited by both the methods applied in the modified conditions indicated the advantage of such way of novel plasma process control.

The idea of using the working gas injection instead of stationary gas flow mode assumes in the first approximation that fast changes in working gas pressure may lead to thermal unequlibrium of

\footnotetext{
${ }^{*}$ This paper was presented at the $5^{\text {th }}$ Congress of the Polish Vacuum Society, Krakow, 12 - 15 September 2013.

${ }^{\dagger}$ E-mail: zdunek@inmat.pw.edu.pl.
}

plasma. Furthermore, the lack of cold gas in the chamber space prior to gas injection makes possible to avoid the kinetic energy dissipation on collision between the plasma particles and the cold gas.

Previously presented initial experimental results [7] have shown the advantages of the proposed modification of the well known methods of plasma surface deposition (in the case of IPD - possibility of exceptionally effective producing of antiabrasive layers with improved morphology on unheated substrates as well as change in target erosion mode during the layer deposition by MS).

However, lately carried out experiments [1, 2] have shown that in the XPS spectra of AlN coatings deposited by GIMS ( as Injection $\underline{\text { Magnetron }}$ Sputtering), higher amount of Al-Al bonds occurred than in the case of the layers obtained in the process using continuous gas flow mode. The appearance of such bonds is probably connected with an undesirable phenomenon called by us " a metallic tail". The reason of that "metallic tail" could be both the diminishing of the portion of reactive gas 
and/or self sputtering effect of the aluminum target, arising during the final stage of the working gas injection. This paper reports on the results of our latest studies on the possibilities of controlling the gas/electric power relation during the GIMS process to eliminate the harmful "metallic tail" effect.

\section{Experimental procedure}

The processes of AlN layers magnetron sputtering deposition were performed in the home made apparatus. As described earlier [1,2] our apparatus was equipped with WMK100 type magnetron supplied by a DPS pulse power supplier operated at a frequency of $100 \mathrm{kHz}$ with $2 \mathrm{kHz}$ modulation. The apparatus allowed us to deposit the layers by the PMS (continuous gas flow) or by our original GIMS technique (gas injection). In the latter case, the continuous flow gas system used during the PMS deposition was replaced by our original gas dosing system for GIMS. This system was equipped with a pulse valve supplied and controlled by an unit specially designed and made by us.

AlN layers produced by GIMS were deposited in two modes: mode A - gas injection in its initial variant as mentioned previously $[1,2]$ i.e. without synchronization of the gas injection/voltage time durations and mode $\mathrm{B}$ - after the just introduced modification of the gas injection procedure. The modification of the gas injection procedure consisted in using a device specially designed by us to control the gas injection/electric power phase relation (patent procedure has been just now initiated and that is why the device could not be described in detail).

AlN layers were deposited on non-heated 2inch Si wafers (GIMS, modes: A and B) or on the Armco plates with a diameter of $20 \mathrm{~mm}$ (PMS). Prior to the AIN layer synthesis the aluminum target of initial thickness of about $8 \mathrm{~mm}$ was preliminary conditioned for $10-15 \mathrm{~min}$ by sputtering in argon (shutter was used) continuously dosed to the chamber. Next, the process of AlN layer deposition was carried out both in the $\mathrm{Ar}+\mathrm{N}_{2}$ atmosphere (PMS, continuous gas flow with the dy- namic pressure of order of $10^{0} \mathrm{~Pa}$ ) or in the nitrogen atmosphere only (GIMS). In the case of GIMS, the nitrogen was injected by a valve pulsing with a frequency of $10^{0}-10^{1} \mathrm{~Hz}$ and the valve time switching-on time duration of order of $10^{-3} \mathrm{~s}$. The pressure of nitrogen was periodically varied depending on the phase of gas injection. However, within an accuracy of the inertia of the pressure meter gauge, the plasma was excited at pressures even as low as $10^{-3} \mathrm{~Pa}$. The final thickness of the layer was dependent on the process time. The synthesis of the AlN layers with the thickness of tens of nanometers (GIMS, Si wafers: SEM, XPS) or about $1 \mu \mathrm{m}$ (PMS, Armco plates: XRD) was carried out at an effective power of several $\mathrm{kW}$, using the ratio between the circulating and effective power as one of essential parameters controlling the course of the plasma process (the circulating power as an important technological parameter results from a special, novel concept of DPS magnetron power supply [11]). Phase composition of the synthesis product was investigated for the thick layers deposited on the Armco substrates with the use of typical XRD technique (Bruker D8). We assumed here that the phase composition of the synthesized layers was not essentially influenced by the gas flow dosing system (continuous vs. injection flow). Morphology of the thin AIN layers deposited on the Si wafers was studied with the SEM (ZEISS Ultra Plus). The AlN layer material obtained by the "conventional" PMS deposition with the continuous working gas $\left(\mathrm{Ar}+\mathrm{N}_{2} / 1 \mathrm{~Pa}\right)$ supply was used as a reference material in the morphology investigation. The distribution of particular bonds, especially $\mathrm{Al}^{2 p}$ orbitals in the material of AlN layers was investigated with the use of the XPS spectroscopy (PHI5700/660 Physical Electronics).

\section{Results and discussion}

A typical XRD spectrum of the relatively thick layers deposited on Armco plates by the PMS is shown in Fig. 1. As might be expected, h-AlN is the product of the synthesis. This nitride phase is typically observed during the layers deposition with the use of magnetron sputtering of an aluminum target [8-10]. 


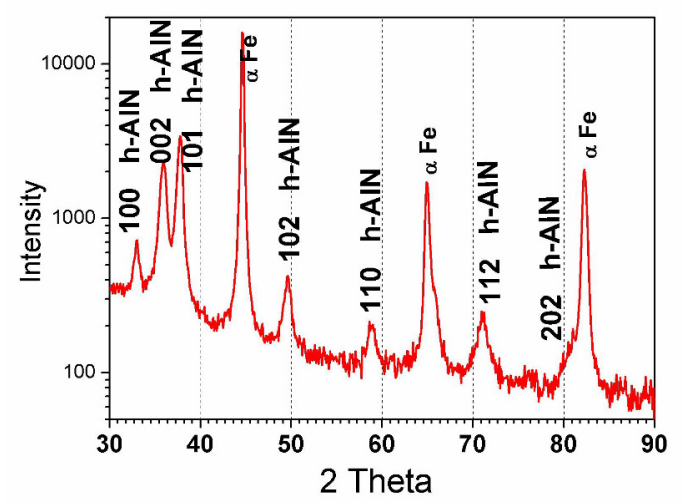

Fig. 1. Phase composition of the layer with the thickness of approximately of $1 \mu \mathrm{m}$, deposited by the PMS.

Fig. 2 presents a comparison of the morphology of a thin AlN layer deposited "conventionally" with the use of the standard PMS and the morphology of an AIN layer deposited by the novel GIMS method. In our opinion, the difference among the structures of both materials seems to be remarkable. Definitely, material of the layer obtained in the GIMS process is characterized by a more favourable morphology. The GIMS AlN layer looks dense, without any traces of columnar structures and the free surface of the layer is smooth.
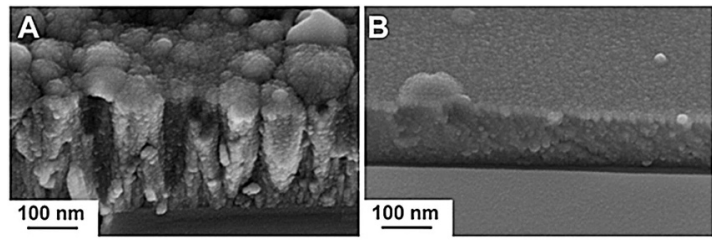

Fig. 2. Morphology of the AlN layers deposited by the PMS (a) and GIMS (b) processes.

Based on our previous experiments related to the synthesis of AlN layers by magnetron sputtering $[1,2]$ we have discovered that in the GIMS conditions the values of forbidden energy band are a bit lower than those measured for the AlN layers deposited by the pulsed magnetron sputtering (4.9 $\mathrm{eV}$ in the case of GIMS layers in comparison to $5.5 \mathrm{eV}$ for layers deposited by the PMS). We have assumed that a possible reason of that is the appearance of the "metallic tail" in the final phase of the discharge, produced by the self-sputtering of aluminum target material.

The presently made XPS studies seem to prove our previous assumption. Fig. 3 presents the spectra of the AlN layers produced in the mode A, i.e. in the same manner as described in the our last paper [2]. Fig. 3a shows the profile of elements distribution in the layer material. The presence of oxygen in the layer material is typical and most probably results from the gas desorption from the chamber walls and air surface adsorption after removing the samples from the chamber and their exposure to the atmosphere. Fig. $3 \mathrm{~b}$ shows the profile analysis of the binding energy for the A12p orbitals. Deconvoluted Al2p peak (Fig. 3c), chosen for the central region of the AlN layer, shows the presence not only of the Al-N bonds characterized by the value of $73.4 \mathrm{eV}$ but also the presence of the $\mathrm{Al}-\mathrm{Al}$ bonds (71.5 eV) [12]. In our opinion, such occurrence can be treated as a strong argument that proves our hypothesis on the presence of self-sputtering in the final stage of discharge ignited by the gas injection during the GIMS working in the mode A.

Taking under consideration the assumption on the self-sputtering in the final stage of the individual gas injection during the GIMS deposition we decided to equip the apparatus control system with a specially constructed controller to control the relation of periods of times of gas injection and the voltage duration in each of the individual gas pulses - mode B in the present experiments.

The XPS results for the AIN layer material produced by the GIMS working in the mode B are presented in the Fig. 4. Similarly to the previous case, the profile of elements distribution seems to be regular, however, a little bit more oxygen has been found for the B mode layer material. As in the previous case, deconvolution of the Al2p peak was made (the spectrum was obtained for the central region of the AlN layer, Fig. 4b). One can see in Fig. $4 c$ that the deconvoluted spectra prove the lack of the "metallic" part of the spectrum and the presence of the Al-N peak with the binding energy of $73.43 \mathrm{eV}$ (in our opinion the presence of the $\mathrm{Al}-\mathrm{O}$ peak with the binding energy of $74.15 \mathrm{eV}$ is not very important from the point view of the matter). 
a)

b)
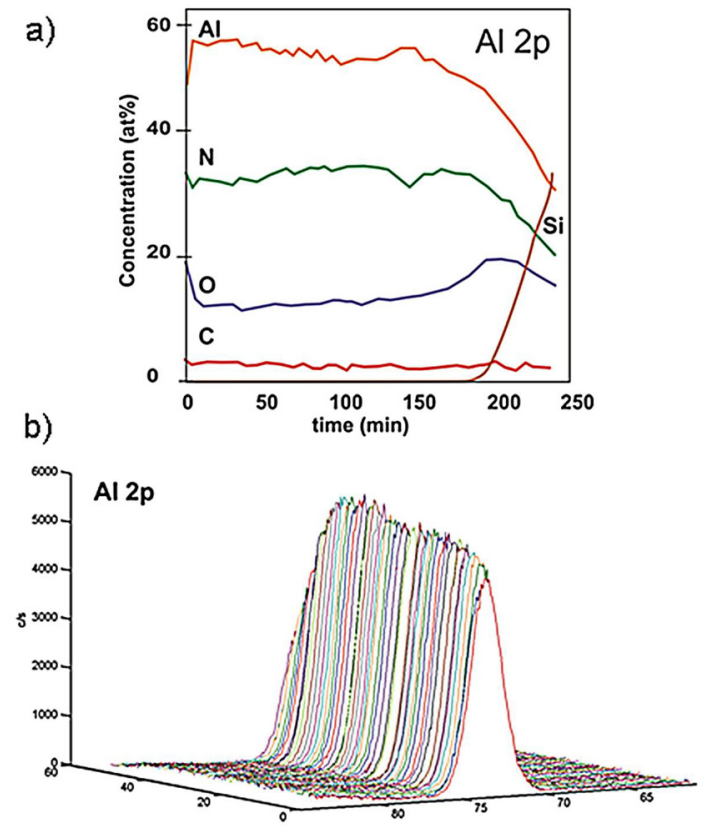

c)

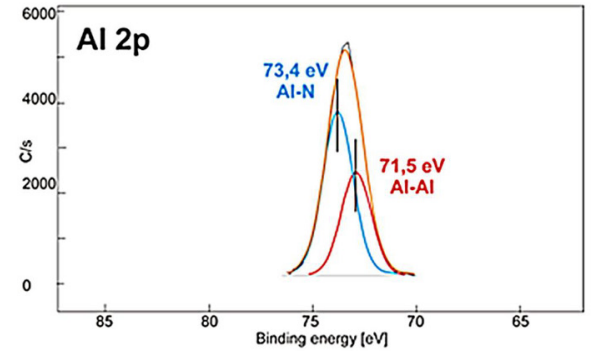

Fig. 3. XPS spectra of the AlN layer produced by the GIMS working in the mode A: a) Profile of elements distribution across the layer, $b$ ) Profile of the binding energy distribution for the Al2p orbitals, c) Deconvolution of the A12p peak proving the existence of $\mathrm{Al}-\mathrm{Al}$ bonds in the layer material.

It is obvious that the results obtained in the present study should be treated as a preliminary stage, and need further, more detailed investigation. Nevertheless, they seem to be very important for the understanding the mechanism of layer deposition by the novel GIMS technique. From our point of view, the precise synchronization of the time duration of the gas impulse in the relation to the time duration of the voltage applied is crucial for the deposition process and finally for the dielectric properties of the AlN layers.

On the other hand, the mentioned above time relation seems to open new prospective possibili- a)

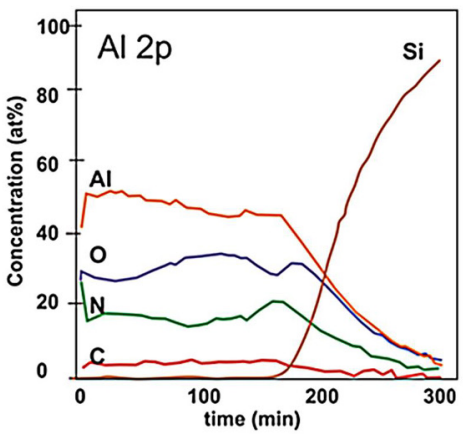

b)

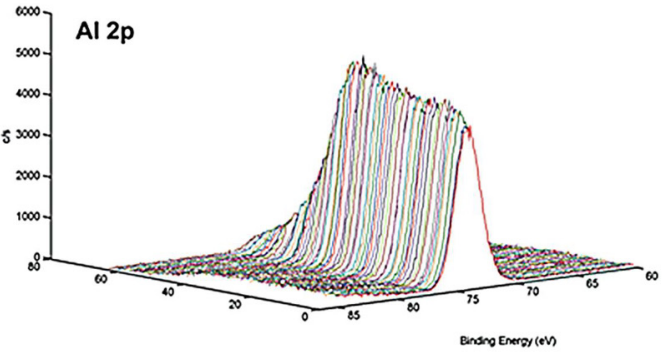

c)

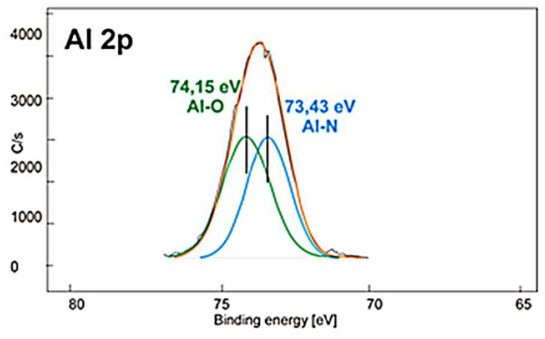

Fig. 4. XPS spectra of the AlN layer produced by the GIMS working in the mode B: a) Profile of elements distribution across the layer, b) Profile of the binding energy distribution for the A12p orbitals, c) Deconvolution of the A12p peak, proving the lack of $\mathrm{Al}-\mathrm{Al}$ bonds in the layer material.

ties to influence the effect of using the GIMS technology for producing the layers with desired phase composition (e.g. metallic/dielectric nanocomposite layers).

\section{Conclusion}

In recent time we have focused our attention on novel magnetron sputtering (MS) technology. The matter consists in using the gas injection as a tool for initiating and effective control of plasma generation process during the layer deposition by magnetron sputtering (PMS $\rightarrow$ GIMS). Based on our 
previous experience in the application of gas injection to control the production of layers by the IPD method, we used a similar technique in the case of MS method. Studies described above concern a successful attempt to gain better control of the plasma process during the magnetron sputtering governed by gas impulse injection. It seems that by applying the specially designed controller, integrated with the electric supply and gas dozing system of the MS apparatus, we can effectively control and even eliminate the tail of metallic vapor (aluminum in the case of the AlN layers) produced in the final stage of each individual gas injection during the GIMS.

\section{Acknowledgements}

The study was financially supported by the National Centre for Research and Development under the Contract No150002-10/10

\section{References}

[1] Zdunek K., Nowakowska-Langier K., Chodun R., Dora J., Gas injection as a tool for plasma process control during coating deposition, invited lecture, AEPSE'2011, Dalian, China, 2011.
[2] Zdunek K., Nowakowska-Langier K., Chodun R., Dora J., Surf. Coat. Tech., 228 (2013), S367.

[3] ZduneK K., Surf. Coat. Tech., 201 (2007), 4813.

[4] Posadowski W.M., Thin Solid Films, 343 - 344 (1999), 85

[5] Posadows KI W.M., Thin Solid Films, 392 (2001), 201.

[6] Arnell R., Kelly P., Bradley J., Surf. Coat. Tech., 188 - 189 (2004), 158.

[7] Zdunek K., Nowakowska-Langier K., Chodun R., KUPCZYK M., SIWAK P., Vacuum, 85 (2010), 514.

[8] Dmitrova V., Manova D., Paskova T., Uzunov T., IVANOV N., DEChEV D., Vacuum, 51 (1998), 161.

[9] Mortet V., Vasin A., Jouan P., Elmazria O., Djouadi M., Surf. Coat. Techn., 176 (2003), 88.

[10] Dallaeva D., Bilalov B., Gitikchiev M., Kardashova G., Safaraliev G., TOMÁneK P., ŠKARVADA P., SMITH S., Thin Solid Films, 526 (2012), 92.

[11] Posadowski W., Wiatrowski A., Dora J., RaDZIMSKI Z., Thin Solid Films, 516 (2008), 4478.

[12] Österle W., Dörfel I., URban I., Reier T., Schultze J. W., Surf. Coat. Techn., 102 (1998), 168.

Received 2013-09-27 Accepted 2014-02-04 